\title{
CHRNE MUTATION AND CONGENITAL MYASTHENIA
}

The CHRNE e1293insG mutation was identified in $14(60 \%)$ of 23 North African families with an early onset form of congenital myasthenic syndrome studied at centers in France, Tunisia, Algeria, and UK. The phenotypic expression was homogeneous with moderate hypotonia and oculobulbar involvement, mild and stable course, and good response to cholinesterase inhibitors. This mutation is thought to originate in an ancient single founder event in the North African population. (Richard P, Gaudon K, Haddad H, et al. The CHRNE 1293insG founder mutation is a frequent cause of congenital myasthenia in North Africa. Neurology Dec 1 2008;71:1967-1972). (Reprints: Dr Daniel Hantai, Inserm U582.47, Boulevard de l'Hopital, Paris, France 75651.

E-mail:d.hantai@institut-myologie.org).

COMMENT. Congenital myasthenic syndrome (CMS) is caused by various genetic defects that include recessive mutations in AChR subunit CHRNE gene. In North Africa, most patients are homozygous for the e1293insG mutation. In Europe, the mutation is associated with a heteroallelic mutation in CHRNE. The DOK7 mutation CMS has a broad clinical phenotype, distinguished by the absence of external ophthalmoplegia, a progressive deterioration of respiratory function, and lack of long-term response to esterase inhibitors (Muller JS et al. Brain 2007;30:1497-1506; Ped Neur Briefs June 2007;21:43-4). Molecular genetic testing is important in diagnosis and therapy of infants with CMS. In patients with COLQ mutations, esterase inhibitors may cause worsening of symptoms (Ped Neur Briefs March 2008;22:17-18). In an early report of 6 infants with a 'neonatal persistent (congenital) myasthenia gravis' treated at the Massachusetts General Hospital, Boston (Millichap \& Dodge. Neurology 1960;10:1007-1014), mothers did not suffer from MG, but 2 cousins of 1 patient and a sister of another were affected. The principal presenting signs were ptosis, weak cry, and generalized weakness. In later childhood, ptosis and external ophthalmoplegia were most prominent, and involvement of bulbar musculature, weakness, and respiratory difficulty were uncommon and mild. Symptoms, especially ophthalmoplegia, were only partially relieved or refractory to pyridostigmine. In the MGH series of pediatric myasthenic patients, 10 infants had the neonatal transient type, 6 a congenital type, and 35 the juvenile type.

\section{LANGUAGE DISORDERS}

\section{AUTOSOMAL DOMINANT PARTIAL EPILEPSY WITH AUDITORY FEATURES}

Auditory and language processing in 17 subjects with autosomal dominant partial epilepsy with auditory features (ADPEAF) was investigated by MRI, fMRI, and MEG and compared to 26 controls, in a study at Columbia University, New York. Age of seizure onset was late childhood or adolescence. MRI revealed no structural abnormality, but fMRI and 
MEG showed reduced or delayed activation patterns in affected subjects. A previous report of a lateral temporal malformation in ADPEAF is not supported by this study, but the findings point to a functional impairment of language processing. (Ottman, R, Rosenberger L, Bagic A, et al. Altered language processing in autosomal dominant partial epilepsy with auditory features. Neurology Dec 9 2008;71:1973-1980). (Reprints: Dr Ruth Ottman, GH Sergievsky Center, Columbia University, 630 West $168^{\text {th }}$ Street, P\&S Box 16, Ne York, NY 10032. E-mail: ro6@columbia.edu).

COMMENT. Autosomal dominant partial epilepsy with auditory features is an idiopathic focal epilepsy syndrome with auditory symptoms or receptive aphasia. Families with this syndrome commonly have mutations in the LGI1 gene. These authors previously published a review of the genetics of 24 ADPEAF families with mutations in the LGI1 gene (Ped Neur Briefs Sept 2008;22:71).

\section{PROGNOSIS OF LANDAU-KLEFFNER SYNDROME}

The long-term follow-up of seven patients with Landau-Kleffner syndrome (LKS) is reported from State University of Campinas, Brazil. All were males between age 8 and 27 years. Parents or patients were interviewed 3 to 16 years after disease onset. All had normal MRIs. All had interictal seizure discharges on the EEG, maximal on the temporal lobes in 5, and exacerbated in sleep. Age of disease onset was 3 to 9 years; 5 patients presented with seizures and 2 with language disorder. Seizures were focal motor in 6 patients; 3 had clonic orofacial movements, 2 had secondarily generalized seizures. Language disorder was insidious in onset in 4 and abrupt in 3. All patients had aphasia and verbal auditory agnosia. Two patients still had seizures at follow-up at 12 and 27 years of age, despite several antiepileptic drugs; seizures had resolved in 4. Language disorder had persisted in 3, it was partially in remission in 3 , and had resolved in 1. Duration of aphasia was 2 to 8 years. One of 4 adult patients is employed, and 3 do not work independently. Three patients are under 13 years of age. Six of 7 patients have severe deficits in communication, and 3 have behavior problems. Only one patient has a normal quality of life. In six with limitations, aphasia/agnosia is the main problem. Shorter duration of aphasia correlates with better outcome. Five patients have normal EEGs, 6-17 years after disease onset; 2 still have abnormal EEGs and refractory seizures. (Duran MHC, Guimaraes CA, Medeiros LL, Guerreiro MM. Landau-Kleffner syndrome: Long-term follow-up. Brain Dev Jan 2009;31:58-63). (Respond: Dr MM Guerreiro. E-mail: mmg@,fcm.unicamp.br). 